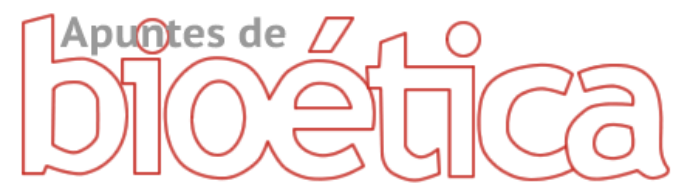

https://doi.org/10.35383/apuntes.v4i1.482

e-ISSN: $2663-4910$

ARTÍCULO DE INVESTIGACIÓN

Universidad Católica Santo Toribio de Mogrovejo

\title{
La necesaria incorporación del farmacéutico comunitario al equipo interdisciplinar que asiste al enfermo en fase terminal
}

\author{
The necessary incorporation of the community pharmacist to the \\ interdisciplinary team that assists the terminally ill patient
}

\author{
Rafael Ibáñez del Pino*, 1, a \\ ribanez.4@alumni.unav.es \\ https://orcid.org/0000-0003-0560-8480
}

\section{Cristina Saiz Rodríguez ${ }^{2, b}$}

csaiz.1@alumni.unav.es

https://orcid.org/0000-0002-1928-9073

\section{Sonia Rivas Borrell ${ }^{3, c}$}

srivas@unav.es

https://orcid.org/0000-0002-8393-3898

José López Guzmán ${ }^{4, d}$

jlguzman@unav.es

https://orcid.org/0000-0001-7308-3087

\author{
* Autor corresponsal \\ ${ }^{1}$ Farmacia Leticia Gago Casanova, Leganés, Madrid, \\ España. \\ ${ }^{2}$ Profesora en Colegio Miravalles-El Redín, Navarra, \\ España. \\ ${ }^{3}$ Docente de la Facultad de Educación y Psicología. \\ Universidad de Navarra, Navarra, España. \\ ${ }^{4}$ Docente de la Facultad de Farmacia. Universidad \\ de Navarra, Navarra, España. \\ a Máster Formación Magistral y Experto en \\ Productos de Parafarmacia \\ ${ }^{\mathrm{b}}$ Licenciada en Educación Primaria \\ c Doctora en Educación \\ ${ }^{\mathrm{d}}$ Doctor en Farmacia
}

Fechas importantes

Recibido: 2020-11-29

Aceptado: 2021-04-09

Publicado online: 2021-07-26

\section{Resumen}

En este trabajo se parte del reconocimiento del importante papel que el farmacéutico comunitario desempeña, en la atención sanitaria, a personas que sufren de un proceso terminal y en sus familiares y/o cuidadores. No hay duda de que es fundamental el consejo, tratamiento y acompañamiento de los profesionales sanitarios en cualquier patología que suponga un compromiso vital. El farmacéutico comunitario, como agente sanitario, debe estar implicado en esos procesos. Sin embargo, en muchas ocasiones es olvidado por un sistema sanitario que desaprovecha, de este modo, a uno de sus profesionales más cualificados.

Estamos convencidos de que el paciente en fase terminal y sus familiares y/o cuidadores se verían muy favorecidos por una implicación mayor de los farmacéuticos en sus procesos. Por esta razón, en 
este artículo se reflexiona sobre los beneficios que reporta la inclusión del farmacéutico comunitario en el equipo asistencial que atiende a las personas en fase terminal.

Palabras clave: Farmacéutico; Enfermo en Fase Terminal; Equipo Asistencial; Farmacia Comunitaria.

\begin{abstract}
This work starts from the recognition of the important role that the community pharmacist plays, in health care, to people who suffer from a terminal process and to their family members and / or caregivers. There is no doubt that the advice, treatment and accompaniment of health professionals is essential in any pathology that involves a vital commitment. The community pharmacist, as a health agent, must be involved in these processes. However, on many occasions it is forgotten by a health system that wastes, in this way, one of its most qualified professionals.

We are convinced that the terminally ill patient and their family members and / or caregivers would be greatly favored by a greater involvement of pharmacists in their processes. For this reason, this article reflects on the benefits of including the community pharmacist in the healthcare team that cares for people in the terminal phase.
\end{abstract}

Keywords: Pharmacist; Terminally ill; Assistance Team; Community Pharmacy.

\title{
Introducción
}

En la atención a enfermos en fase terminal se acostumbra a mantener que deben ser cuidados por un equipo asistencial que resuelva satisfactoriamente sus variadas necesidades. Del mismo modo, es habitual hacer una diferencia en ese equipo asistencial, estableciendo que existe un equipo básico, formado por médicos y enfermeras, y otro subsidiario integrado por distintos profesionales que trabajan en colaboración con el equipo básico. Entre los miembros de ese equipo subsidiario se puede encontrar una variada gama de profesionales: especialistas en el duelo, capellanes, dietistas, especialistas en linfedema, terapeutas ocupacionales, oncólogos, farmacéuticos, fisioterapeutas, psiquiatras, psicólogos, trabajadores sociales, logopedas, etc. Frente a esa visión generalizada de profesionales de distinto rango, cada vez hay más voces que reclaman, en beneficio del paciente, una mayor integración de todo el equipo sanitario. Ese loable objetivo solo será posible valorando a los profesionales por su grado de responsabilidad en cada proceso, no fundamentando el equipo en una trasnochada distribución estratificada de funciones basada en el grado universitario que se ha cursado. En este marco, se hace necesario subrayar que el papel del farmacéutico comunitario, en la atención a enfermos en fase terminal y/o a sus familiares y cuidadores, debería cobrar mayor importancia debido a que es el especialista del medicamento. Sin embargo, se acostumbra a olvidar al farmacéutico, algo inconcebible por ser, como ya se ha indicado, el experto en el medicamento. El desplazamiento del farmacéutico de la primera 
línea de la asistencia sanitaria también se ha evidenciado en la pandemia de covid, cuando los farmacéuticos ni tan siquiera han sido mentados, en muchas ocasiones, al hacer referencia a los agentes sanitarios. No obstante, los farmacéuticos han estado en primera línea durante la pandemia, junto a los otros agentes sanitarios, facilitando la medicación, proporcionando tratamientos off-label, o elaborando nuevas dosificaciones de medicamentos comercializados para otras patologías.

En este trabajo se parte del reconocimiento del importante papel que el farmacéutico comunitario desempeña, en la atención sanitaria, a personas que sufren de un proceso terminal y en sus familiares y/o cuidadores. No hay duda de que es fundamental el consejo, tratamiento y acompañamiento de los profesionales sanitarios en cualquier patología que suponga un compromiso vital, como pueden ser el cáncer, VIH, Alzheimer, etc. El farmacéutico comunitario, como agente sanitario, debe estar implicado en esos procesos pero, como se ha indicado en el párrafo anterior, en muchas ocasiones es olvidado por un sistema sanitario que desaprovecha, de este modo, a uno de sus profesionales más cualificados.

Estamos convencidos de que el paciente en fase terminal y sus familiares y/o cuidadores se verían muy favorecidos por una implicación mayor de los farmacéuticos en sus procesos. Por esta razón, vamos mostrar los beneficios que reporta la inclusión del farmacéutico comunitario en el equipo sanitario y los aspectos que deberían ser favorecidos para la consecución de este fin.

\section{Desarrollo}

1. Contribución del farmacéutico comunitario en la atención a pacientes en fase terminal.

\subsection{Adecuación del tratamiento}

El farmacéutico es el experto en el medicamento, es el profesional que conoce los principios activos, los excipientes que los acompañan, las formas farmacéuticas en las que son comercializados, la manera de liberarse, absorberse, distribuirse, metabolizarse y excretarse el medicamento en el organismo, etc. (Domenech, Martínez y Plá, 1997). De ahí, que el farmacéutico suponga una garantía en el control de que el medicamento prescrito por el médico sea el adecuado en el momento de la elección, y lo siga siendo en la evolución del proceso a través de una correcta farmacovigilancia. Por lo tanto, es necio desperdiciar la labor del 
farmacéutico relegando su labor a la lectura de unas recetas y la venta de los medicamentos en ellas consignados, cuando su implicación más directa puede ser fundamental en la asistencia del paciente.

\subsection{Conciliación del tratamiento}

En los últimos años se está otorgando una gran importancia al tema de la conciliación de los medicamentos debido a que el enfermo en fase terminal suele estar sometido a una polifarmacia (en unas ocasiones determinada por la patología desencadenante del proceso terminal y, en otras, coadyuvada por otros procesos) que hay que controlar para que haya una adherencia, para evitar incompatibilidades, repeticiones, etc. Al menos la mitad de los errores de medicación se producen cuando cambia el responsable médico del paciente; por esta razón, en las transiciones asistenciales el paciente es especialmente vulnerable a este tipo de errores (Delagado-Sánchez et al., 2009). Una manera de minimizar estas equivocaciones es realizar en cada transición asistencial (ingreso en el hospital, cambio de servicio, alta hospitalaria...) una correcta conciliación de la medicación. Existen muchas definiciones de conciliación. Para Delgado "es el proceso formal que consiste en valorar el listado completo y exacto de la medicación previa del paciente juntamente con la prescripción farmacoterapéutica después de una transición asistencial, al ingreso en el hospital, después de un cambio del responsable médico o al alta hospitalaria" (Villamayor, Herrero, de Miguel, Bouzas y Freire, 2016). El objetivo del proceso de conciliación de la medicación es garantizar que los pacientes reciben todos los medicamentos necesarios que estaban tomando previamente, asegurándose de que están prescritos con la dosis, la vía y la frecuencia correctas, y que son adecuados a la situación del paciente y a la nueva prescripción. También intenta evitar las interacciones y duplicidades entre la medicación crónica y la aguda, ajustándose a la situación clínica actual (Paez et al., 2010).

Está claro que la implicación del farmacéutico en esos procesos redundaría en beneficio del paciente, de los otros agentes sanitarios, y de la misma sociedad. Según la Sociedad Española de Cuidados Paliativos (SECPAL) "no son necesarios más de 15 o 20 medicamentos para controlar los síntomas de los pacientes en fase terminal, de los que será necesario tener un conocimiento exhaustivo. De esta manera, se evitará el ir cambiando de un medicamento a otro e ir probando 
por sistema los últimos que salen al mercado" (Flor, Pérez, Hortz, Díaz y Raja, 2005). A la hora de utilizar un fármaco en un paciente en fase terminal será fundamental valorar la relación riesgo/beneficio, o lo que es lo mismo, su efecto terapéutico y sus efectos secundarios. Actividad que, como hemos dicho anteriormente, estará en buenas manos con un farmacéutico en el equipo. Como bien indica Newton, el control del enfermo en fase terminal por parte del farmacéutico "es la última oportunidad de un paciente para una terapia farmacológica segura y efectiva" (Newton, 2016).

El farmacéutico comunitario puede contribuir a paliar las discrepancias que se producen entre los medicamentos que toma el paciente, no hay que olvidar que estos problemas no solo hay que abordarlos en el ingreso hospitalario (Saavedra, et al., 2016), sino que también son latentes después del alta hospitalaria porque el paciente puede estar tomado los medicamentos prescritos en el hospital y, además, otros prescritos por médicos privados o recomendados por personas ajenas al ámbito sanitario (no deja de ser habitual que, ante procesos terminales, se recurra a distintas terapias e incluso a productos milagro en un intento desesperado de encontrar una mejora o solución).

\subsection{Control de las interacciones}

Hay que tener en cuenta que la aparición de efectos secundarios es la causa más frecuente de abandonos del tratamiento (Flor et al., 2005). De ahí que no es raro que, cada vez más, se insista en la importancia del farmacéutico en el control de las interacciones de medicamentos en la farmacología que trata a pacientes en el final de sus días. Según Morgan et al. "a medida que se acerca la muerte, los pacientes están en su punto más frágil, pero una carga de síntomas cada vez mayor suele requerir un aumento de los medicamentos, lo que los pone en mayor riesgo de interacciones farmacológicas" (Morgan, Rowett y Currow, 2015). Este mayor uso de medicamento incrementa la posibilidad de interacciones. No obstante, hay que tener presente que algunas de esas interacciones serán inevitables. Sin embargo, otras interacciones podrán ser evitadas y es ahí donde recobra un importante papel el farmacéutico.

Una vez reconocido que hay que evitar las interacciones innecesarias y que el farmacéutico está en buena disposición, la mejor, para llevar a cabo ese papel, habría que plantear cual sería la estrategia más conveniente para acometerlo. En 
este punto ya hay discrepancias, algunos autores mantienen que se necesita más investigación para esa determinación. Es evidente que el farmacéutico podría defender más su papel, cuantificando su actividad y proporcionando datos que confirmen lo que beneficia su colaboración más activa en los equipos de cuidados paliativos o de atención a enfermos en fase terminal. Sin embargo, se han realizado muy pocas investigaciones rigurosas sobre la contribución del farmacéutico en la reducción de la utilización de medicamentos innecesarios en adultos mayores frágiles o pacientes que se acercan al final de la vida y, por ello, el uso excesivo de medicamentos innecesarios en adultos mayores frágiles con una esperanza de vida limitada sigue siendo un desafío poco estudiado (Tjia et al., 2013). A pesar de esa falta de más evidencias, ya hay investigadores que se pronuncian, reconociendo que es "probable que los servicios de cuidados paliativos necesiten implementar múltiples estrategias, incluido un mayor uso de software de prescripción computarizado y un mayor vínculo más cercano con los farmacéuticos clínicos" (Morgan et al, 2015). De la misma forma, es previsible que el farmacéutico comunitario aporte un valor añadido al equipo de atención del enfermo en fase terminal controlando las posibles interacciones de los medicamentos, tanto las previsibles como las no esperadas y observadas o deducidas en el diálogo con el enfermo.

\subsection{Cercanía al paciente}

Se ha demostrado que el paciente en fase terminal se encuentra más cómodo en su casa, rodeado de su familia y sus recuerdos, que en el hospital. Se hace alusión a que el hogar se convierte en su zona de confort para transitar el último periodo de su vida (Santos, 2014). Un buen cuidado en el hogar requiere de un equipo multidisciplinar integrado en el que se otorgue una relevancia a la presencia de un profesional farmacéutico y no se desdeñe el papel de los familiares (Canga, Canga, García-Vivar, 2016). Para lograr este objetivo es preciso valorar las diferentes cuestiones logísticas a la hora de atender a enfermos en fase terminal en sus domicilios.

Cuando hacemos alusión a una participación multidisciplinar, creemos que debe hacerse una matización en lo que respecta a la esencia de dicho equipo. Abogamos por el equipo interdisciplinar frente al multidisciplinar. Así, según Francesc Torralba "es preciso distinguir aquí entre interdisciplinariedad y 
multidisciplinariedad. El primer término indica una relación fecunda entre disciplinas distintas, un diálogo continuado y complementario, mientras que el segundo término se refiere a la mera yuxtaposición formal de materias" (Torralba, 1998). Mientras que en el equipo multidisciplinar cada disciplina aporta su saber para resolver un problema, en el interdisciplinar no sólo lo aporta, sino que trata de integrarse con las demás disciplinas para establecer un objetivo compartido. Se establece un diálogo verdadero de intercambio de información, definiendo una realidad común para entenderse y que no haya un desfase de comprensión. Si el médico, la enfermera, el psicólogo, el trabajador social, el farmacéutico y todos los miembros del equipo, hablan un lenguaje común comprensible e integrado es más fácil aunar esfuerzos y conseguir buenos resultados (Delgado, 2005).

El cuidado del enfermo en el hogar puede tener un gran impacto para los cuidadores familiares. Entre los principales problemas identificados se encuentran la pérdida del empleo o reducción de las horas destinadas al cuidado de la persona enferma (Tallman, Greenwald, Reidenouer y Pantel, 2012), el agotamiento físicoemocional (Rabow, Hauser y Adams, 2004), la aparición de enfermedades (Strachan, Ross, Rocker, Dodek y Heyland, 2009), la falta de un afrontamiento efectivo de la enfermedad, la falta de apoyo social (Seidman, Pano, Dorina, Bail y Acrich, 2004), el escaso tiempo para sí mismo (Hanratty, et al., 2012), y el aumento de la dependencia del rol de cuidador (Hasson et al., 2010). A pesar de que el cuidado del enfermo genera numerosos beneficios a sus cuidadores (Rivas, 2015), no hay duda de que estos cuidadores familiares necesitan de protección y ayuda de distinto tipo: técnica, emocional, etc. Los miembros del equipo asistencial deben estar pendientes de ello y convertirse en un elemento de ayuda y, por supuesto, nunca suponer una nueva carga generando nuevos problemas. El farmacéutico comunitario tiene una relación directa y cercana, en muchos casos de confianza, con esos familiares y es un valioso elemento de ayuda, siempre que se le integre en el equipo asistencial y se le reconozca su grado de responsabilidad en el proceso asistencial.

Con respecto al seguimiento en el hogar, por parte de un profesional sanitario, la evidencia ha mostrado que favorece el manejo de la medicación y el control de los síntomas, disminuye la incertidumbre del paciente y cuidador, facilita la discusión en torno a los temas del final de la vida, brinda asesoramiento nutricional, ayuda con el manejo de equipos técnicos y ofrece a las personas una sensación de 
seguridad y supervisión en las labores del cuidado (Arias, García-Vivar, 2015). La capacitación de los cuidadores y pacientes a domicilio debe planificarse de manera que se ajuste a los horarios y cubra todos los detalles, como el funcionamiento del dispositivo de infusión, la documentación y a quién contactar en caso de cambios en la condición del paciente. Además de la capacitación, los cuidadores necesitan apoyo psicosocial. En el enfoque interdisciplinario de la atención domiciliaria, farmacéuticos, enfermeras, sicólogos, terapeutas físicos y ocupacionales, trabajadores sociales y otros participan en un equipo de atención domiciliaria. Los familiares y cuidadores a menudo asumen gran parte de la responsabilidad de evaluar el estado del paciente, informar al médico y coordinar los cambios en la terapia. El entorno del hogar exige políticas y procedimientos flexibles y métodos simples y concisos de monitoreo y documentación, especialmente cuando se maneja el dolor intratable.

En las citadas estrategias que se están adoptando hay que destacar el reconocimiento de estándares de práctica para el cuidado en el hogar. Con su aplicación se ha demostrado que la atención domiciliaria es una alternativa racional para muchos pacientes con enfermedades terminales, pero que plantea nuevos desafíos (Norwood, 1990). Como, por ejemplo, que no exista una comunicación efectiva entre el equipo de atención hospitalario y los profesionales del ámbito comunitario, dificultad en el manejo de ciertas situaciones en el hogar, escaso entendimiento entre los diferentes niveles de atención, confusión en el reconocimiento de los roles profesionales y el rango de servicios prestados por las instituciones de salud $u$ ofrecidos en la comunidad (Arias, 2015). No obstante, el número de pacientes con enfermedades crónicas y lentamente debilitantes, como enfermedades cardíacas y enfermedades pulmonares en etapa terminal, está en aumento y que optan por pasar los últimos días de su vida en su hogar y no en un hospital. Por este motivo, los sistemas de atención médica necesitan expandirse para acomodarse a esa nueva situación asistencial. Es cierto que el número de programas formales de cuidados paliativos está incrementándose rápidamente, con el crecimiento más documentado para pacientes hospitalizados pero, por desgracia, los programas de cuidados paliativos en el hogar son menos comunes y habrá que fomentarlos para obtener datos constatables que permitan su diseminación y aplicación (Renee, 2007). Esta nueva situación requiere de una respuesta firme por parte del profesional sanitario, que deberá adaptar su trabajo 
para lograr una mejoría de calidad de vida de estos pacientes. Aquí, por supuesto, también tiene un papel primordial la figura del farmacéutico comunitario, como profesional sanitario cercano y con una preparación acorde para sustentar un papel intermediario en el tránsito del paciente desde el hospital a su domicilio. Una vez más, nos preguntamos si todos los conocimientos del farmacéutico están de verdad al servicio del paciente, o si éstos están siendo infrautilizados porque el farmacéutico se ha acomodado a la labor comercial de sus farmacias comunitarias, o porque otros profesionales sanitarios, o la misma administración sanitaria, los han relegado a un segundo nivel en la asistencia sanitaria.

\subsection{Benefico farmaeconómico}

En la actualidad, los recursos que pueden ser destinados al gasto sanitario son limitados, por lo que es necesario racionalizar su consumo y priorizar en la asignación de estos recursos, atendiendo a criterios de equidad y justicia. Los estudios de farmacoeconomía permiten conocer cuál es la eficiencia de las diferentes alternativas terapéuticas disponibles en el mercado para tratar las distintas enfermedades, $y$, por lo tanto, ayudan a determinar qué opciones terapéuticas deberían emplearse de forma rutinaria (Soto, 2001). También permiten dilucidar cuál va a ser la relación coste/efectividad de las alternativas terapéuticas farmacológicas existentes en el mercado para tratar las distintas enfermedades. En este sentido, ofrecen información complementaria a la eficacia, seguridad y calidad que proporcionan los programas de investigación clínica (Soto, Fernández, 1998).

Por lo indicado en el anterior párrafo, la inclusión en los formularios de las opciones terapéuticas más eficientes redundará en un mejor uso de los recursos disponibles, y en un aumento de la calidad asistencial. En este sentido, ya se ha evidenciado que si se muestra a los agentes decisores el uso de los medicamentos más eficientes, se favorece una mejor redistribución de los recursos existentes en el hospital. Del mismo modo, en el ámbito hospitalario se ha demostrado que la incorporación de los medicamentos, que presenten una mejor relación coste/efectividad y/o calidad de vida (Soto, 2001), a los protocolos y guías de práctica clínica hospitalaria son un factor favorable en el control de los recursos. Estas evaluaciones son realizadas por el farmacéutico que, de esta manera, colabora de manera eficaz en la optimización de los recursos. Este modelo 
hospitalario puede trasladarse al comunitario ya que, al existir tanta polimedicación en los enfermos en fase terminal, la farmacovigilancia por parte del profesional del medicamento puede colaborar con la farmacoeconomía, por ejemplo, evitando duplicidad de la mediación, medicación innecesaria, ajuste posológico actualizado, etc.

\section{Elementos a considerar para favorecer la integración del farmacéutico comunitario en la atención a pacientes en fase terminal.}

Una vez se ha demostrado que el farmacéutico comunitario, si es correctamente integrado en el equipo sanitario, puede favorecer notablemente la asistencia a enfermos en fase terminal en atención domiciliaria, así como a sus cuidadores, ya sean del entorno familiar o de tipo profesional, pasamos a reflexionar sobre los elementos que pueden favorecer o dificultar la integración del farmacéutico comunitario en ese equipo sanitario asistencial domiciliario.

\subsection{Estructuras que favorezcan la integración}

La integración del farmacéutico, como parte efectiva del equipo sanitario que atiende al paciente en fase terminal en su domicilio, requiere de cambios conceptuales y estructurales de distinta índole. En primer lugar, se tiene que producir un cambio legal que favorezca el reconocimiento de la farmacia comunitaria como un establecimiento de atención primaria que pueda coordinarse con los demás centros de atención primaria. Un reconocimiento que para ser efectivo debe comenzar con la posibilidad de compartir la historia clínica del paciente, con todas las garantías que ofrece la confidencialidad y el respeto de la autonomía del paciente. Es un contrasentido que distintos profesionales sanitarios atiendan a un mismo paciente con distinto conocimiento de la evolución de su patología y de los tratamientos que utiliza.

La segunda cuestión que es preciso reformular es la de las competencias de las distintas profesiones sanitarias, ya se ha indicado anteriormente que hay que crear estructuras que fomenten la auténtica interdisciplinariedad. Las profesiones evolucionan para responder a las necesidades de los pacientes y supone un contrasentido que ese desarrollo se vea coartado o limitado por culpa del corporativismo de algunos profesionales. 


\subsection{Adquisición de habilidades complementarias}

Se ha indicado en párrafos anteriores que ante nuevas situaciones se requiere de nuevos planteamientos estructurales y también de formación. En este sentido, si el farmacéutico comunitario quiere adoptar un papel más activo en la atención a pacientes en fase terminal tendrá que estar más preparado en aquellas cuestiones, principalmente farmacológicas, que afectan a este grupo de población. No obstante, la formación no solo debe quedar circunscrita a aquella de tipo técnico y farmacológico. El paciente en fase terminal es muy vulnerable y, por ello, requiere que los profesionales que los atienden adquieran otras habilidades dirigidas a su cuidado integral. Por ejemplo, hay dos habilidades que el farmacéutico deberá adquirir, porque la mayoría no la ha recibido en su formación académica, como son unos fundamentos de sicología y de comunicación.

En una gran parte de las Facultades de Medicina y Enfermería se ha incluido una asignatura de introducción a la Psicología que, junto con la Antropología, le permite al alumno, entender mejor al paciente. Consideramos que el farmacéutico comunitario que quiere realizar bien su trabajo con enfermos en fase terminal deberá adquirir esos conocimientos, si no los ha recibido ya en el grado.

En cuanto a la comunicación, es fácilmente comprensible que tener buenas habilidades de comunicación es un prerrequisito esencial para proporcionar cuidados paliativos de calidad e influye positivamente en la calidad de vida del paciente. Cuando pensamos en los cuidados a una persona, siempre o casi siempre, imaginamos acciones positivas donde nuestros actos se materialicen de una forma real en el bienestar del paciente. Nuestra idea del cuidado suele ser procurar su alimentación, hidratación, descanso, etc. Pero normalmente se nos olvida la importancia que tiene la comunicación en dicho cuidado, pudiendo ser la misma comunicación una forma de cubrir las necesidades de la persona enferma o afectada (Delgado, 2005).

Cuando se alude al trabajo ordinario del farmacéutico se acostumbra a desdeñar que, en ocasiones, las herramientas más importantes son la palabra y la escucha. El establecer una comunicación abierta con el enfermo en fase terminal es para los cuidadores un escollo difícil de salvar en la práctica diaria. La muerte y el proceso de morir evocan en nosotros reacciones psicológicas que llevan, directa o indirectamente, a evitar la comunicación. Nunca es fácil contestar a preguntas 
como: ¿Cuánto me queda de vida? ¿Cómo voy a morir? ¿Por qué a mí? Ante este tipo de preguntas el profesional no preparado se queda sin respuesta (Buisán, Delgado, 2007).

Esa situación de falta de herramientas del farmacéutico en el diálogo con el enfermo en fase terminal motiva que se requiera, durante la formación del farmacéutico, cierta atención a su adiestramiento en comunicación con el paciente. En conclusión, la atención farmacéutica al paciente en fase terminal y/o a sus familiares $y / 0$ cuidadores debe tener un enfoque amplio, en el que se tenga en consideración una visión holística del sujeto y se atienda a sus necesidades terapéuticas sin olvidar el contexto emocional, social y espiritual. En este contexto, el farmacéutico deberá adquirir el necesario conocimiento científico y técnico, y también las habilidades comunicativas necesarias para saber transmitir esa información tan sensible al paciente.

\section{Conclusiones}

Cada vez es más habitual que personas con procesos terminales quieran pasar los últimos días de su vida en sus domicilios. De ahí que la asistencia sanitaria tenga que sufrir un desplazamiento desde el ámbito hospitalario al domiciliario. En este escenario, ese preciso que se cuente con el farmacéutico comunitario ya que es el profesional sanitario más próximo al domicilio, y suele mantener una relación de cercanía y/o confianza con el paciente, la familia y sus cuidadores. Para esa integración en el equipo asistencial domiciliario se deberán acometer algunos cambios estructurales (legales) y conceptuales (optar por la interdisciplinariedad) en cuanto a la conformación de las profesiones sanitarias. Al mismo tiempo, el farmacéutico comunitario se deberá preocupar en adquirir algunas competencias como, por ejemplo, habilidades en comunicación.

\section{Referencias}

Arias, M., García-Vivar, C. (2015). La transición de cuidados paliativos del hospital al hogar: una revisión narrativa de las experiencias de pacientes y cuidadores familiares. Invest Educ Enferm, 33(3): 482-491

Buisán, R., Delgado, J.C. (2007). El cuidado del paciente terminal. Anales del Sistema Sanitario de Navarra, 30 (Suppl.3): 103-112. 
Canga, A., Canga, N., García-Vivar, C. (2016). Enfoque sistemático familiar: necesidad de formación para los profesionales de la salud. En: A.M. González, P. Arregui, y C Montoro (Eds.). Familia y Sociedad en el siglo XXI (pp.95-102). Madrid: Dykinson.

Delgado, J.C. (Coord.) (2005). Los cuidados del confort en el final de la vida: Responsabilidad enfermera. Asociación de Bioética Fundamental y Clínica.

Delgado-Sánchez, O., Nicolás-Picó, J., Martínez-López, I., Serrano-Fabiá, A., Anoz-Jiménez, L., Fernández-Cortés, F. (2009). Errores de conciliación en el ingreso y en el alta hospitalaria en pacientes ancianos polimedicados. Estudio prospectivo aleatorizado multicéntrico. Medicina Clínica, 133:741-744.

Domenech, J., Martínez, J., Plá, J.M. (1997). Biofarmacia y Farmacocinética. Madrid: Síntesis.

Flor, A., Pérez, I., Hortz, A., Díaz, A., Raja, M.I. (2005). Tratamiento farmacológico en los cuidados paliativos. Boletín Farmacoterapéutico de Castilla-La Mancha, VI(2): 1-8.

Hanratty, B., Holmes, L., Lowson, E., Grande, G., Addington-Hall, J., Payne, S., et al. (2012) Older adults' experiences of transitions between care settings at the end of life in England: a qualitative interview study. J Pain Symptom Manage, 44 (1):74-83.

Hasson, F., Kernohan, W.G., McLaughlin, M., Waldron, M., McLaughlin, D., Chambers, H., et al. (2010). An exploration into the palliative and end-of-life experiences of carers of people with Parkinson's disease. Palliat Med, 24(7):731-736.

Morgan, N.A., Rowett, D., Currow, D.C. (2015). Analysis of drug interactions at the end of life. BMJ Supportive \& Palliative Care, 5: 281-286

Newton, D.W. (2016). Does Your Drug Expertise Include Clinical Pharmaceutics? Int J Pharm Compd, 20(3): 202-206.

Norwood, M. (1990). Home care of the terminally ill. Am J Hosp Pharm, 47 (8 Suppl):S23-26.

Páez-Vives, F., Recha-Sancho, R., Altadill-Amposta, A., Montaña-Raduá, R.M., Anadón-Chortó, N., Castells-Salvadó, M. (2010). Abordaje interdisciplinar de la conciliación de la medicación crónica al ingreso en un hospital. Revista de Calidad Asistencial, 25(5):308-313.

Rabow, M.W., Hauser, J.M., Adams, J. (2004). Perspectives on care at the close of life. Supporting family caregivers at the end of life: "they don't know what they don't know". JAMA, 291(4):483-491.

Renee Hill, R. (2007). Clinical pharmacy services in home-based clinical palliative care program. American Journal of Health-System Pharmacy, 64 (8): 806-810.

Rivas, S. (Coord.)(2015). Generaciones conectadas: beneficios educativos derivados de la relación entre nietos y abuelos. Madrid: Pirámide.

Saavedra-Quirós, V., Montero-Hernández, E. et al. (2016). Conciliación de la medicación al ingreso y alta hospitalaria. Una experiencia consolidada. Revista de Calidad Asistencial, 31 (51): 45-54.

Santos, J. (2014). El derecho a morir en casa. Reflexiones de un médico. Revista de Calidad Asistencial, 29 (5): 291. 
Seidman, S., Pano, C.O., Dorina, S., Bail, V., Acrich, L. (2004) Sentimiento de sobrecarga y apoyo social en cuidados familiares de enfermos crónicos. Revista de Psicología, 22 (1): 45-62.

Soto, J., Fernández, P. (1998). Evaluación económica de medicamentos: aplicaciones prácticas para los diferentes agentes decisores. Medicina Clinica, 110: 699-702.

Soto, J. (2001). Estudios de Farmacoeconomía ¿por qué, cómo, cuándo y para qué? Medifam, 11(3): 147.

Strachan, P.H., Ross, H., Rocker, G.M., Dodek, P.M., Heyland, D.K., Canadian Researchers at the End of Life Network (CARENET).(2009) Mind the gap: Opportunities for improving end-of-life care for patients with advanced heart failure. Can J Cardiol, 25(11):635-640

Tallman, K., Greenwald, R., Reidenouer, A., Pantel, L. (2012). Living with advanced illness: longitudinal study of patient, family, and caregiver needs. Perm J, 16(3):28-35.

Tjia, J., Velten, S.J., Parsons, C. et al. (2013). Studies to Reduce Unnecessary Medication Use in Frail Older Adults: A Systematic Review. Drugs Aging, 30, 285-307.

Torralba i Rosselló, F. (1998). Antropología del cuidar. Madrid: Fundación Mapfre.

Villamayor-Blanco, L., Herrero-Puch, L., de Miguel Bouzas, J.C., Freire-Vázquez, M.C. (2016). Conciliación de medicamentos al ingreso mediante un programa de prescripción electrónica asistida. Farmacia Hospitalaria, 40 (5): 334.

\section{Cómo citar este trabajo}

Ibañez del Pino, R., Saiz Rodríguez, C., Rivas Borrell, S., \& López Guzmán, J. (2021). La necesaria incorporación del farmacéutico comunitario al equipo interdisciplinar que asiste al enfermo en fase terminal. Apuntes De Bioética, 4(1), 88-101. https://doi.org/10.35383/apuntes.v4i1.482

\section{Financiación}

El presente artículo no cuenta con financiación específica para su desarrollo y/o publicación.

\section{Agradecimientos}

Innovation Factory de la Universidad de Navarra.

\section{Conflicto de interés}

Los autores del artículo declaran no tener ningún conflicto de intereses en su realización.

C Los autores. Este artículo es publicado por la Revista Apuntes de Bioética del Instituto de Bioética, Universidad Católica Santo Toribio de Mogrovejo.

Este es un artículo de acceso abierto, distribuido bajo los términos de la Licencia Creative Commons Atribución-NoComercial-CompartirIgual 4.0 Internacional (CC BY-NC-SA 4.0), que permite el uso no comercial, distribución y reproducción en cualquier medio, siempre que la obra original sea debidamente citada. 